“드 2013 IEEE. Personal use of this material is permitted. Permission from IEEE must be obtained for all other uses, in any current or future media, including reprinting/republishing this material for advertising or promotional purposes, creating new collective works, for resale or redistribution to servers or lists, or reuse of any copyrighted component of this work in other works." 


\title{
Model Predictive Control of Permanent Magnet Synchronous Machine with Reduced Torque Ripple
}

\author{
Hongming $\mathrm{Hu}^{1 *}$, Jianguo $\mathrm{Zhu}^{2}$, Youguang $\mathrm{Guo}^{2}$ \\ ${ }^{1}$ Department of Electrical Engineering, Wuhan University of Technology, China \\ ${ }^{2}$ Faculty of Engineering and Information Technology, University of Technology, Sydney, Australia \\ E-mail: Hongming.Hu@uts.edu.au
}

\begin{abstract}
The conventional direct torque control (DTC) method features fast dynamic response, but it also has notable drawbacks such as high torque and flux ripples, variable switching frequency and acoustic noise. The new DTC scheme based on model predictive control (MPC) is studied in the paper for permanent magnet synchronous machine drive. The paper aims to reduce the torque and flux ripple. Firstly oneand two-step delay MPC methods are employed with or without three torque/flux and switching frequency conditions. Furthermore linear extrapolations N-step delay MPC method is taken into account. Compared to simulation results, the twostep delay DTC scheme is better than the one-step scheme in the steady response and dynamic performance, at the same time the one the torque and frequency compensations can reduce switching frequency from high value to a low constant one. Simulation results are presented to validate the effectiveness of the proposed schemes in this paper.
\end{abstract}

\section{INTRODUCTION}

Permanent magnet synchronous machine (PMSM) drives are nowadays widely used due to its low inertia, high efficiency, high power/torque density and high reliability, and are considered superior to induction motor and DC motor. For the applications requiring high dynamic performance, field oriented control (FOC) is usually employed, which requires relatively complex rotary transformation and accurate rotor position. The dynamic response is limited by the bandwidth of inner current loop.

Conventional DTC directly manipulates the final output voltage, and hence eliminates the delay and features a high dynamic response [1-2]. For DTC, all calculations are implemented in stationary coordinate, so the structure is simple. Despite the merits above, conventional switchingtable-based DTC presents some notable drawbacks, such as high torque and flux ripples, variable switching frequency and acoustic noise [3-5]. To address these problems, a number of methods have been proposed in the literature, of which the space vector modulation (SVM) is usually employed [6]. The SVM based DTC provides low torque ripple and fixed switching frequency. However, it requires rotary coordinate transformation, knowledge of many motor parameters and high computational ability; these negate the simplicity and robustness of the conventional DTC.

Apart from SVM-DTC, the predictive control method can combine more control variables in one cost function, such as torque and flux, and control them at the same time. The cost function is employed to weight the effects of different voltage vectors and select the one which minimizes the cost function. The cost function employed MPC features its flexibility in incorporating any nonlinear constraints that can be modeled mathematically, which can decrease torque and flux ripples [717].

Another kind of approach is to introduce the duty cycle control in conventional DTC. By adjusting the duty ratio of the active vector from the switching table, the torque ripple can be reduced significantly.

In this paper, the model based predictive torque control (MPTC) is introduced to overcome this problem . The principle of model predictive control is to predict the future behavior of the variables over a time frame based on the model of the system . All predictions are evaluated based on a cost function to find the voltage vector which results in the minimum cost.

\section{MACHINE EQUATIONS OF PMSM}

For a PMSM, the model in the rotor synchronous coordinate is the most popular, because all the parameters become constant. The machine equations of PMSM in the synchronous frame are expressed as follows.

$$
\begin{aligned}
& u_{d}=R_{s} i_{d}+L_{d} \frac{d i_{d}}{d t}-\omega L_{q} i_{q} \\
& u_{q}=R_{s} i_{q}+L_{q} \frac{d i_{q}}{d t}+\omega L_{d} i_{d}+\omega \psi_{f} \\
& \psi_{d}=L_{d} i_{d}+\psi_{f} \\
& \psi_{q}=L_{q} i_{q}
\end{aligned}
$$

The electromagnetic torque is expressed as below.

$$
\begin{aligned}
& T_{e}=\frac{3}{2} p\left(\psi_{d} i_{q}-\psi_{q} i_{d}\right) \\
& =\frac{3}{2} p\left(\psi_{f} i_{q}+\left(L_{d}-L_{q}\right) i_{d} i_{q}\right) \\
& =\frac{p_{q}\left|\psi_{s}\right|}{2 L_{d} i_{q}}\left[2\left(\psi_{f} L_{q} \sin \delta+\left(L_{d}-L_{q}\right)\left|\psi_{s}\right| \sin 2 \delta\right)\right]
\end{aligned}
$$

where

$u_{s}$ stator voltage vector

$R_{s}$ stator resistance

$i_{s}, i_{d}, i_{q}$ stator current vector, d-axis and q-axis component

$\omega$ rotor speed

$\psi_{s}$ stator flux vector 


\author{
$L_{d}, L_{q} \mathrm{~d}$-axis and q-axis inductance \\ $\psi_{f}$ permanent magnet flux \\ $T_{e}$ electromagnetic torque \\ $p$ number of pole pairs
}

\section{MODEL PERDICTIVE CONTROL ON PMSM}

\section{A. Basic principle}

A DC/AC inverter is employed in the main circuit, which outputs six effective voltage vectors and two naught voltage vector as shown in Fig. 1. In conventional DTC [2], hysteresis comparators and switching table are used to select the appropriate voltage vector. The principle of model predictive control is to predict the future behavior of the variables over a time frame based on the above model of the system. All predictions are evaluated based on a cost function to find the voltage vector which results in the minimum cost. In the improved DTC control diagram [1] as shown in Fig. 3 the cost function is selected in the case which both torque and flux is as close as possible to the commanding value at end of the cycle. In the paper the cost function is defined as

$$
\begin{aligned}
& g=\left|T_{e}^{*}-T_{e}\right|+k\left(\left|\psi_{s}^{*}\right|-\left|\psi_{s}\right|\right) \\
& u_{s} \in\left\{\mathbf{V}_{0}, \mathbf{V}_{1}, \ldots \mathbf{V}_{6}, \mathbf{V}_{7}\right\}
\end{aligned}
$$

where $k$ is the weighting factor. In this paper, torque and flux are given the same weight, so $k$ is selected as $T_{n} / \varphi_{n}$, where $T_{n}$. and $\varphi_{\mathrm{n}}$ are the rated value for the torque and stator flux. The MPTA is the maximum value of torque/current, expressed as below

$$
\varphi_{\mathrm{s}}=\frac{\mathrm{T}_{\mathrm{e}}}{\mathrm{pi}_{\mathrm{s}}}
$$

Torque and flux are calculated according to formulae (3), (4) and (5).

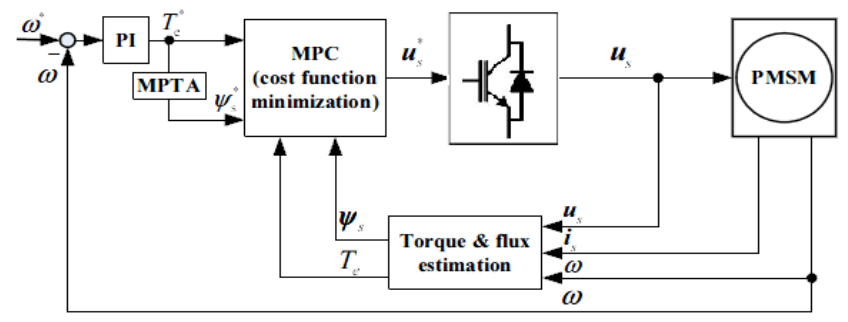

Fig. 1. An improved DTC control diagram

\section{B. Principle of Model Predictive Control}

The $2 \mathrm{~L}$-VSI converter can be defined as the determination of an appropriate control action $S(\mathrm{i})(\mathrm{i}=1-8)$ that will drive a generic system variable $x(t)$ as close as possible to a desired reference value $x^{*}(t)$. Consider the qualitative behaviour [3] of $x(t)$ and its regularly sampled value $x\left(t_{\mathrm{k}}\right)$ over a sample period
$T_{s}$ for a system with a finite number of control actions $n$, as shown in Fig. 4. They can be evaluated together with the measured value $x\left(t_{\mathrm{k}}\right)$, based on a prediction function $f$, to predict all the possible system transitions $x_{i}\left(t_{k+1}\right)=f\left\{x\left(t_{k}\right), S_{i}\right\}$, for $\mathrm{i}=1, \ldots, 8$. To determine which of the control actions is to be selected, a cost function $f$ is used, which is usually dependent on absolute error between the predictions and the reference $g_{i}=\left|x^{*}\left(t_{k+1}\right)-x_{i}\left(t_{k+1}\right)\right|$. Here variable $x$ represents torque or flux.

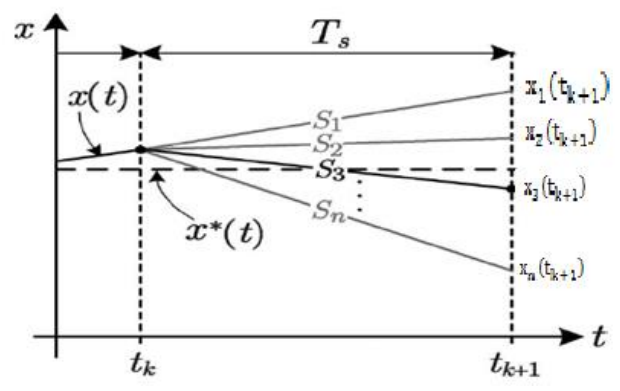

Fig. 2. One-step delay MPC operating principle

Combining (1) and (2), the PMSM machine model can be rewritten in terms of state pace equations ${ }^{[4]}$ as

$$
\begin{array}{r}
\frac{d i_{d}}{d t}=\frac{-R_{S} i_{d}+\omega L_{q} i_{q}+u_{d}}{L_{d}} \\
\frac{d i_{q}}{d t}=\frac{-R_{S} i_{q}-\omega L_{d} i_{d}+u_{q-\omega \varphi_{f}}}{L_{q}}
\end{array}
$$

The equations of PMSM in (11) and (12) are used to obtain a prediction of the stator current at the next sampling instant, $i_{s}^{k+1}$, based on a given stator voltage $\boldsymbol{u}_{s}^{k}$ and measured current $i_{s}^{k}$ at current sampling instant. The prediction is expressed as

$$
\begin{gathered}
i_{d}^{k+1}=i_{d}^{k}+\frac{1}{L_{d}}\left(-R_{s} i_{d}^{k}+\omega^{k} L_{q} i_{q}^{k}+u_{d}^{k}\right) T_{s} \\
i_{q}^{k+1}=i_{q}^{k}+\frac{1}{L_{q}}\left(-R_{s} i_{q}^{k}-\omega^{k} L_{d} i_{d}^{k}+u_{q}^{k}-\omega^{k} \varphi_{f}\right) T_{s}
\end{gathered}
$$

After obtaining $i_{s}^{k+1}$, both the torque and flux at the $(\mathrm{k}+1)$ th instant can be obtained from (3) (4) and (5).

As it is well known that torque and flux are important variable, a cost functioncan be defined as below.

$$
\begin{aligned}
& g=\left|T_{e}^{*}-T_{e}^{k+1}\right|+k\left(\left|\psi_{s}^{*}\right|-\left|\psi_{s}^{k+1}\right|\right) \\
& u_{s}^{k} \in\left\{\mathbf{V}_{0}, \mathbf{V}_{1}, \ldots \mathbf{V}_{6}, \mathbf{V}_{7}\right\}
\end{aligned}
$$

The coefficient $\mathrm{k}$ can be decided theoretically by the weight between torque and flux, which can be modified flexibly by MATLAB simulation [5][6].

\section{B. Consideration of Two Step Delay}

In (13) it is the one-step delay to define the cost function.From (11) and (12) the variable $i_{d}^{k+1}$ and $i_{q}^{k+1}$ at the $(\mathrm{k}+1)$ th instant is derived from the variable $i_{d}^{k}, \omega^{k}, i_{q}^{k}, u_{d}^{k}$ together with the constant variable $R_{s}, L_{q}, L_{d}, \varphi_{f}$ and ${ }^{T_{s}}$.From Fig.2 $i_{d}$ and ${ }^{i_{q}}$ can be obtained by abc/dq transformation. 


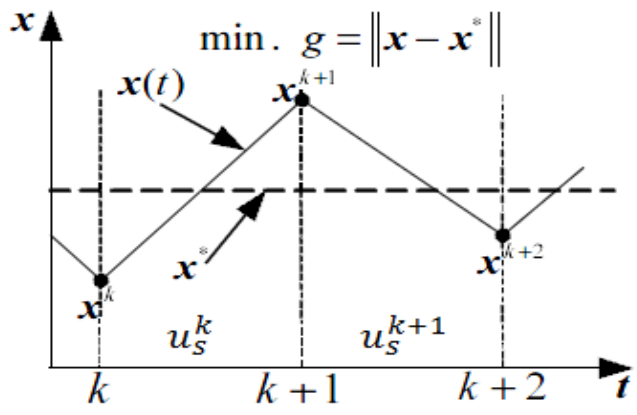

Fig. 3. Two-step delay in digital control system

Considering the calculation of one-step delay, the cost function in (6) should be changed to (14) as shown below very necessary for predictive control in digital implementation. That is to say Two-step delay in digital control system:

$$
\begin{aligned}
\min & g=\left|T_{\varepsilon}^{*}-T_{e}^{k+2}\right|+k\left(\left|\boldsymbol{\psi}_{s}^{*}\right|-\left|\boldsymbol{\psi}_{s}^{k+2}\right|\right) \\
\text { s.t } & \boldsymbol{u}_{s}^{k+1} \in\left\{\mathbf{V}_{0}, \mathbf{V}_{1}, \ldots \mathbf{V}_{6}, \mathbf{V}_{7}\right\}
\end{aligned}
$$

To obtain $T_{\varepsilon}^{k+2}$ and $\psi_{s}^{k+2}$ in (14), a two-step prediction is required. $\boldsymbol{i}_{s}^{k+1}$ is firstly obtained from (11) and (12) and then in a similar way, $\boldsymbol{i}_{s}^{k+2}$ can be predicted with estimated $\boldsymbol{i}_{s}^{k+1}$ as new initial state and $\boldsymbol{u}_{s}^{k+1}$ as input using (11) and (12). Knowing $\boldsymbol{i}_{s}^{k+2}, T_{\varepsilon}^{k+2}$ and $\psi_{s}^{k+2}$ can be obtained from (3) ,(4) and (5). All the possible vector from $V_{0}$ to $V_{7}$ for $\boldsymbol{u}_{s}^{k+1}$ will be evaluated to obtain the one minimizing (14).

C. Consideration of N Step Delay

The expression of the proposed cost function is

$$
\begin{gathered}
\text { min. } g=\left|T_{\varepsilon}^{*}-T_{e}^{k+2}\right|+k\left(\left|\psi_{s}^{*}\right|-\left|\psi_{s}^{k+2}\right|\right) \\
+A\left(\left|T_{\varepsilon}^{*}-T_{\varepsilon}^{k+N}\right|+k\left(\left|\psi_{s}^{*}\right|-\left|\psi_{s}^{k+N}\right|\right)\right) \\
\text { s.t. } \boldsymbol{u}_{s}^{k+1} \in\left\{\mathbf{V}_{0}, \mathbf{V}_{1}, \ldots \mathbf{V}_{6}, \mathbf{V}_{7}\right\}
\end{gathered}
$$

the stator flux $T_{\varepsilon}^{k+N}$ and torque $\psi_{s}^{k+N}$ at $(k+N) t h$ instant are predicted from the value at $(k+1)$ th and $(k+2) t h$ instants using linear extrapolations, which are expressed as

$$
\begin{aligned}
T_{\varepsilon}^{k+N} & =T_{\varepsilon}^{k+1}+(N-1)\left(T_{e}^{k+2}-T_{e}^{k+1}\right) \\
\left|\psi_{s}^{k+N}\right| & =\left|\psi_{s}^{k+1}\right|+(N-1)\left(\left|\boldsymbol{\psi}_{s}^{k+2}\right|-\left|\boldsymbol{\psi}_{s}^{k+1}\right|\right)
\end{aligned}
$$

Where $\mathrm{N}$ larger than 2.

D. Consideration of reduced switching frequency

One of the well-known disadvantages of the hysteresis based DTC scheme are variable high switching frequency. One of the merits of the cost-function based predictive control is its flexibility in incorporating nonlinear constraints. In this section, the switching frequency reduction will be modelled and combined into the cost function based on (9).

The expression of the item is as follows.

$$
\begin{aligned}
& \qquad g_{f}=\sum_{i=a, b, c}\left|u_{s}^{k+1}\left(S_{i}\right)-u_{s}^{k}\left(S_{i}\right)\right| \\
& \text { s.t. } \boldsymbol{u}_{s}^{k+1} \in\left\{\mathbf{V}_{0}, \mathbf{V}_{1}, \ldots \mathbf{V}_{6}, \mathbf{V}_{7}\right\}
\end{aligned}
$$

the item is related to commutation frequency reduction, where $\boldsymbol{u}_{s}^{k}\left(S_{i}\right)$ and $\boldsymbol{u}_{s}^{k+1}\left(S_{i}\right)$ represent the switching state of phase i(i $=\mathrm{a}$, $\mathrm{b}, \mathrm{c})$ at the present cycle (during kth and $(\mathrm{k}+1)$ th instant) and the next cycle (during $(\mathrm{k}+1)$ th and $(\mathrm{k}+2)$ th instant), espectively.

\section{SIMULATION RESULT}

To validate the proposed strategy, simulations using Matlab/Simulink are carried out. The system and machine parameters are listed in Tab. I, II, which are referenced to [12].

TABLE I

MOTOR AND SYSTEM PARAMETERS

\begin{tabular}{|l|l|l|}
\hline Number of pole pairs & $p$ & 3 \\
\hline Permanent magnet flux & $\psi f$ & $0.1057 \mathrm{~Wb}$ \\
\hline Stator resistance & $R s$ & $1.8 \Omega$ \\
\hline d-axis and q-axis inductance & $L d, L q$ & $15 \mathrm{mH}$ \\
\hline Rated speed & $n_{N}$ & $2000 \mathrm{rpm}$ \\
\hline Rated torque & $T_{N}$ & $4.5 \mathrm{Nm}$ \\
\hline Rated line-line voltage & $U_{N}$ & $128 \mathrm{~V}$ \\
\hline Sampling period & $T_{s}$ & $100 \mu \mathrm{s}$ \\
\hline DC bus voltage & $U_{d c}$ & $200 \mathrm{~V}$ \\
\hline control parameters & $\mathrm{k}$ & 25.6 \\
\hline
\end{tabular}

The performances of model predictive control are comparatively investigated in this section by using simulations in the environment of Simulink.

A. Steady Responses and dynamic performance

Firstly the steady responses and dynamic performance is tested in Fig4-6. The paper presents the chatacteristics for One-step delay DTC and two-step delay DTC. From top to bottom, the curves shown in Fig4-6 are the current of phase a,speed,torque and stator flux respectively. In the section
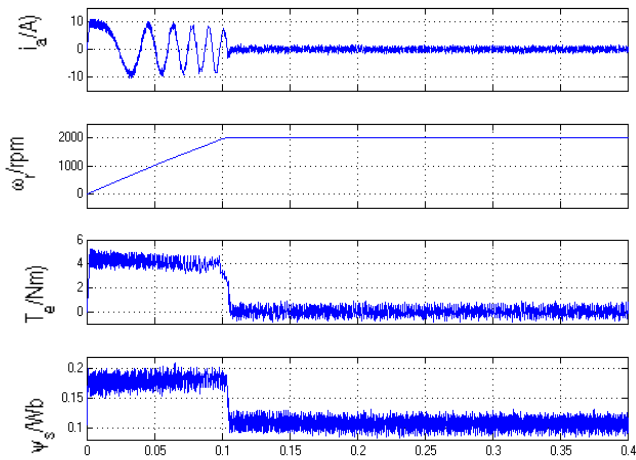

(a)
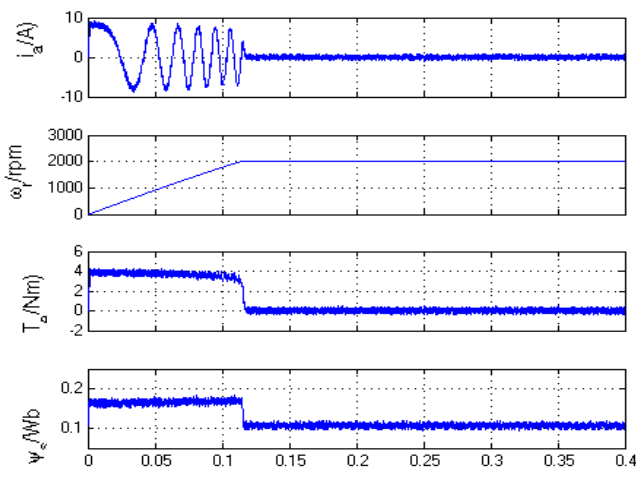

(b)

Fig. 4. Steady responses at rated speed without load:(a) One-step delay DTC, (b) Two-step delay DTC

The motor starts from standstill to $100 \%$ rated speed without load, There are lower torque and flux ripples for two-step delay DTC without compensation shown in Figs. 4 compared to One-step delay DTC. 
(a)
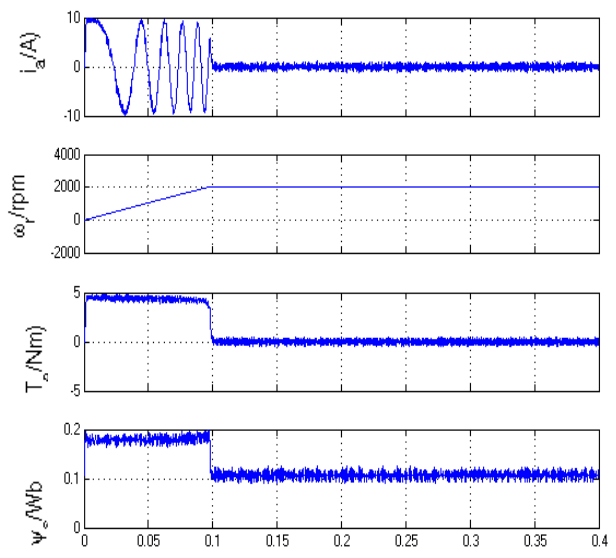

(b)
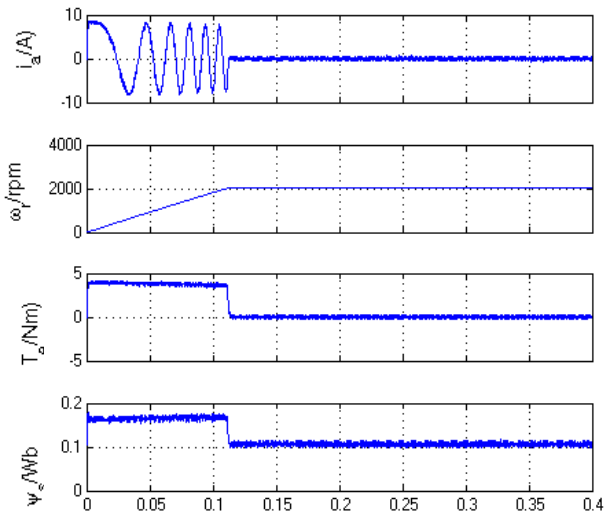

Fig. 5. Steady responses at rated speed without load for MPTC with torque

(a) One-step delay DTC compensation

Under the above same condition, there are lower stator current,torque and flux ripples for two-step delay DTC without compensation shown in Figs. 5 compared to One-step delay DTC.

(a)
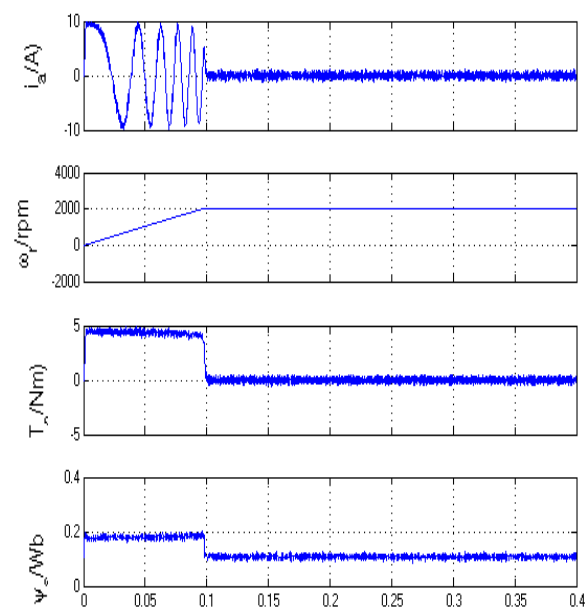

(b)
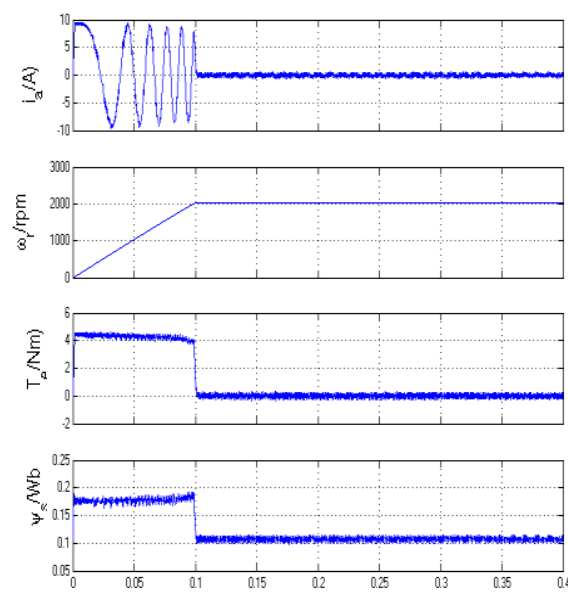

Fig. 6. Steady responses at rated speed without load for MPTC with torque and frequency compensation

$\begin{array}{ll}\text { (a) One-step delay DTC } & \text { (b) two-step delay DTC }\end{array}$

Under the above same condition, there are lower stator current, torque and flux ripples for two-step delay DTC without compensation shown in Figs. 6 compared to One-step delay DTC further.

B. Consideration of switching frequency

Figs. 7 to 8 illustrate the switching frequency characteristics without compensation, with torque compensation and with torque compensation and frequency compensation, espectively. Fig. 7 presents Switching characteristics of One-step delay DTC in the above three conditions. (a)
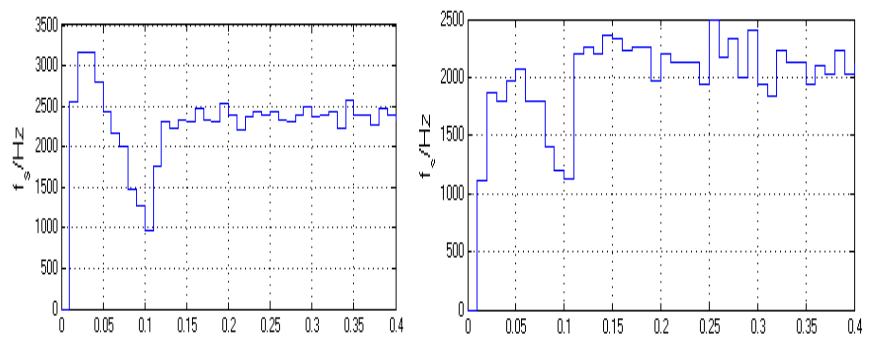

(c)

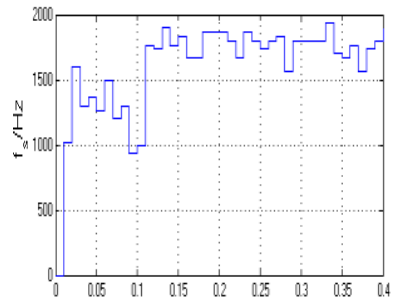

Fig. 7. Switching characteristics of One-step delay DTC method using cost function in (13);

(a)without compensation (b) with torque compensation;

(c) with torque compensation and frequency compensation

There are lowest switching frequency with torque compensation and frequency compensation, highest without compensation under the condition of One-step delay DTC method.

(a) 

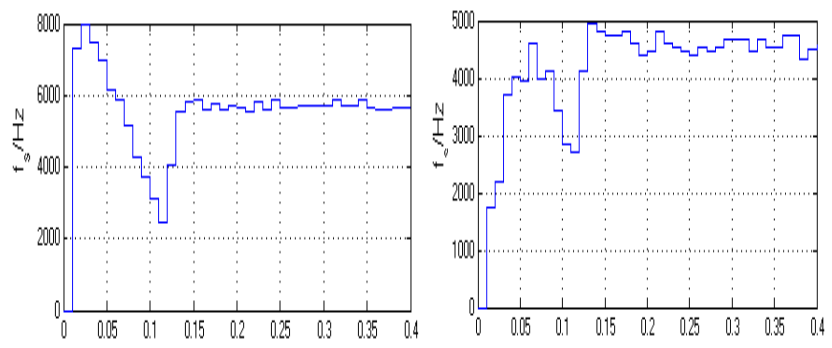

(c)

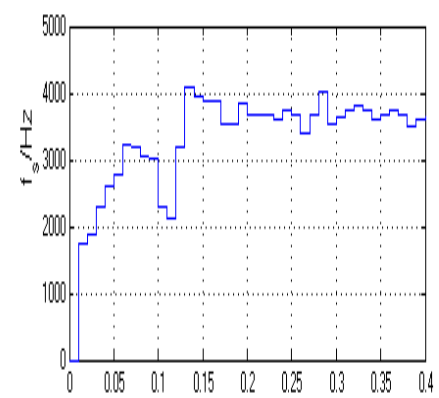

Fig. 8. Switching characteristics of two-step delay DTC method using cost function in (13);

(a)without compensation (b) with torque compensation;

(c) with torque compensation and frequency compensation

There are lowest switching frequency with torque compensation and frequency compensation, highest without compensation under the condition of two-step delay DTC method.The results are the same as of one-step delay DTC method.

\section{CONCLUSION}

A novel model based predictive torque control method is studied in this paper. Some cost functions are studied to get better dynamic performance and reduce the torque ripple. Simulation results can be summarized as follows:

1) The two-step delay DTC scheme is better than one-step delay DTC in the steady response and dynamic performance.

2) The two-step delay DTC scheme features lower torque and flux ripples than the one-step delay.

3) In the two-step delay DTC scheme the torque and frequency compensations are employed can furtherly optimize, at the same time reduce switching frequency from high value to a low constant one.

\section{REFERENCES}

[1] Yongchang Zhang, Jianguo Zhu, and Wei Xu, "Predictive torque control of permanent magnet synchronous motor drive with reduced switching frequency," in Proc. Int. Conf. on Electrical Machines and Systems (ICEMS), 2010, pp. 798-803.

[2]M. Depenbrock, "Direct self-control (DSC) of inverter-fed induction machine," IEEE Trans Power Electron., vol. 3, no. 4, pp. 420-429, 1988.

[3]I. Takahashi and T. Noguchi, "A new quick-response and high-efficiency control strategy of an induction motor," IEEE Trans. Ind. Appl., vol. IA22, no. 5, pp. 820-827, 1986.

[4]L. Zhong, M. F. Rahman, W. Y. Hu, and K. W. Lim, "Analysis of direct torque control in permanent magnet synchronous motor drives," IEEE Trans Power Electron., vol. 12, no. 3, pp. 528-536, 1997.

[5] Thomas J. Vyncke, Ren'e K. Boel, and Jan A.A. Melkebeek, "Direct torque control of permanent magnet synchronous motors - an overview," in Proc. $3^{\text {rd }}$ IEEE Benelux Young Researchers Symposium in Electrical Power Engineering, 27-28 April 2006, Ghent, Belgium, pp. 28- 32.

[6] D. Swierczynski and M. P. Kazmierkowski, "Direct torque control of permanent magnet synchronous motor (PMSM) using space vector modulation (DTC-SVM)-simulation and experimental results,", in Proc. IEEE 28th Annual Conference of the Industrial Electronics Society, 2002, pp. 751-755.

[7] S. Kouro, P. Cortes, R. Vargas, U. Ammann, and J. Rodriguez, "Model predictive control - a simple and powerful method to control power converters," IEEE Trans. Ind. Electron., vol. 56, no. 6, pp. 1826-1838, Jun. 2009.

[8] Tianshi Wang, Youguang Guo, Jianguo Zhu, and Yongchang Zhang, "An assessment of the influence of sampling frequency on steady-state performance of PMSM drive system," in Proc. 22 ${ }^{\text {nd }}$ Australasian Univ. Power Eng. Conf., 2012, pp. 1-4.

[9] Y. Zhang, J. Zhu, and W. Xu, "Analysis of one step delay in direct torque control of permanent magnet synchronous motor and its remedies," in Proc. Int. Conf. Electrical Machines and Systems, 2010, pp. $792-797$.

[10]Yongchang Zhang, Jianguo Zhu, and Wei Xu, "Predictive torque control of permanent magnet synchronous motor drive with reduced switching frequency," in Proc. Int. Conf. Electrical Machines and Systems, 2010, pp. 798-803.

[11] Yongchang Zhang and Jianguo Zhu, "A novel duty cycle control strategy to reduce both torque and flux ripples for DTC of permanent magnet synchronous motor drives with switching frequency reduction," IEEE Trans. Power Electron., vol. 26, no. 10, pp. 3055-3067, 2011.

[12] Yongchang Zhang and Jianguo Zhu, "Direct torque control of permanent magnet synchronous motor with reduced torque ripple and commutation frequency," IEEE Trans. Power Electronics, vol. 26, no. 1, pp. 235-248, 2011.

[13] Yongchang Zhang, Jianguo Zhu, Wei Xu, and Youguang Guo, "A simple method to reduce torque ripple in direct torque-controlled permanent-magnet synchronous motor by using vectors with variable amplitude and angle," IEEE Trans. Ind. Electron., vol. 58, no. 7, pp. 2848-2859, 2011.

[14] Yongchang Zhang, Jianguo Zhu, Zhengming Zhao, Wei Xu, and D. G. Dorrell, "An improved direct torque control for three-level inverter-fed induction motor sensorless drive," IEEE Trans. Power Electron., vol. 27, no. 3, pp. 1502-1513, 2012.

[15] Yongchang Zhang, Zhengming Zhao, Jianguo Zhu, Wei Xu, and D. G. Dorrell, "Speed sensorless direct torque control of 3-level inverter-fed induction motor drive based on optimized switching table," inProc. IEEE 35th Annual Industrial Electronics Conference, pp. 1316-1321. 